\title{
Cooperative Error Handling and Shallow Processing
}

\author{
Tanya Bowden \\ Computer Laboratory \\ University of Cambridge \\ Pembroke St. \\ Cambridge CB2 3QG \\ U. K. \\ Tanya.Bowden@cl.cam.ac.uk
}

\begin{abstract}
This paper is concerned with the detection and correction of sub-sentential English text errors. Previous spelling programs, unless restricted to a very small set of words, have operated as post-processors. And to date, grammar checkers and other programs which deal with ill-formed input usually step directly from spelling considerations to a full-scale parse, assuming a complete sentence. Work described below is aimed at evaluating the effectiveness of shallow (sub-sentential) processing and the feasibility of cooperative error checking, through building and testing appropriately an error-processing system. A system under construction is outlined which incorporates morphological checks (using new two-level error rules) over a directed letter graph, tag positional trigrams and partial parsing. Intended testing is discussed.
\end{abstract}

Unless a keyboard user is particularly proficient, a frustrating amount of time is usually spent backtracking to pick up mis-typed or otherwise mistaken input. Work described in this paper started from an idea of an error processor that would sit on top of an editor, detecting/correcting errors just after entry, while the user continued with further text, relieved from tedious backtracking. Hence 'co-operative' error processing. But if a program is to catch such errors very soon after they are entered, it will have to operate with less than the complete sentence.

Work underway focuses on shallow processing: how far error detection and correction can proceed when the system purview is set to a stretch of text which does not admit complete sentential analysis. To date, grammar checkers and other programs which deal with illformed input usually step directly from spelling considerations to a full-scale sentence parse. However treating the sentence as a basic unit loses meaning when the 'sentence' is incomplete or illformed. Shallow processing is also interesting because it should be cheaper and faster than a complete analysis of the whole sentence.

To investigate issues involved in shallow processing and cooperative error handling, the pet (processing errors in text) system is being built. The focus is on these two issues; no attempt is being made to produce a complete product ${ }^{1}$. Pet operates over a shifting window of text (it can be attached simply and asynchronously to the Emacs editor). One word in this purview is in focus at a time. Pet will give one of three responses to this word; it will accept the word, suggest a correction, or indicate that it found an error it couldn't correct. Below follow an outline and discussion of the (linguistic) components of pet and discussion of testing and evaluation of the system.

\section{Pet System}

\section{Morphological Processing \& Spelling Checking}

The word in focus is first passed through a two level morphological analysis stage, based on an adaption of (Pulman, 1991). Two purposes are served here: checking the word is lexical (i.e. in the lexicon or a permissible inflection of a word in the lexicon) and collecting the possible categories, which are represented as sets of feature specifications (Grover, 1993).

This morphological lookup operates over a character trie which has been compressed into a (directed) graph. Common endings are shared and category information is stored on the first unique transition. The advantages of this compression are that (1) a word/morpheme is recognised (and category affixation rules (Grover, 1993) checked) as soon as the initial letters allow uniqueness, rather than at the end of the word, and (2) there is an immense saving of space. There was a reduction of over half the transitions on the trie formed from the Alvey lexicon.

If the word is unknown, the system reconsiders analysis from the point where it broke down with

\footnotetext{
${ }^{1}$ In particular, there are many $\mathrm{HCI}$ issues associated with such a system, which are beyond the scope of this paper.
} 
the added possibility of an error rule. There are currently four error rules, corresponding to the four Damerau transformations: omission, insertion, transposition, substitution (Damerau, 1964) - considered in that order (Pollock, 1983). The error rules are in two level format and integrate seamlessly into morphological analysis.

$$
\text { * }-\mathrm{X} \text { - * } \rightarrow{ }^{*} \ldots \text {. . }
$$

This says that any letter (' $X$ ') can be inserted, with asterisks indicating that it can occur in any context (compare with (Pulman, 1991)). The right hand side represents the 'error surface' and the left hand side the surface with error removed.

If this doesn't succeed, it backtracks to try an error rule at an earlier point in the analysis. At present it will not apply more than one error rule per word, in keeping with findings on error frequencies (Pollock, 1983).

As an alternative, a program was developed which uses positional binary trigrams (Riseman,1974) (p.b.t.'s) to spot the error position and to check candidate corrections generated by reverse Damerau transformations. This should have the advantage over the two level error rules in that it uses a good method of calculating likely error positions and because a set of correction possibilities can be generated fairly cheaply. (Correction possibilities are ranked using frequency information on Damerau errors and by giving preference to very common words.) However initial tests over a small file of constructed errors showed that the error rules did just as well (slightly better in fact) at choosing the 'correct correction'.

The error rules are applied when ordinary morphological rules fail - which is usually a place p.b.t.'s would mark as in error - but the rules don't ignore error locations p.b.t.'s accept as allowable letter combinations. Most importantly, the error rules operate over a letter graph of the lexicon, so only ever consider lexical words (unknown letters are instantiated to the letters associated with the transition options). The disadvantage remains that generating many correction possibilities (with SICStus backtracking) is time-consuming. At present this phase postulates only one grapheme at a time, although all its possible categories are passed along together to later stages. If all of these categories eventually fail analysis, backtracking to alternative correction candidates (different graphemes) will occur.

\section{Tag Checking \& Partial Parsing}

The Alvey features are mapped on to the CLAWS tagset used in the LOB corpus (Garside, 1987). Tag transitions are checked against an occurrence matrix of the tagged LOB corpus using positional binary trigrams similar to those used in the spelling checks mentioned above. Tag checks though the current set of categories stop when one category passes, but backtrack and continue if parsing then fails.

The Core Language Engine (CLE) is an application independent, unification based "general purpose device for mapping between natural language sentences and logical form representations" (Alshawi, 1992). Its intermediate syntactic stages involve phrasal parsing followed by full syntactic analysis (top-down, left-corner). If the latter stage fails, CLE invokes partial parsing.

The phrasal phase and partial parsing have been extracted and are being adapted to the present purpose. After mapping onto CLE tags, application of the phrasal phase, which implements bottom-up parsing, is straightforward. CLE partial parsing, using left-corner analysis combined with top-down prediction on the results of the phrasal phase, looks for complete phrases and breaks down a wordstring into maximal segments.

(a) the the brown bear $\rightarrow$ the $\mid$ the brown bear (b) ate the nice friendly $\rightarrow$ ate $\mid$ the $\mid$ nice $\mid$ friendly

For example, (a) produces 1 segment and (b) produces 4 segments - whereas "ate the nice friendly cat" would produce 1 segment.

Partial parsing needs to be adapted to support the idea of the pet purview; partial parsing that accepts any string likely to constitute part of a sentence. To achieve this the ends of the wordstring delimited by the purview need to be treated differently. On the right hand end, 'can start rule' possibilities of words can be considered, using the prediction facility already built into the parsing process. The left hand side could be treated by 'can end' possibilities, but a better idea should be to keep within the purview ('remember') previously derived constituents that involve current words.

There is a phase to be added after detection of a tag or partial parsing error. Currently processing will just backtrack to the intraword correction level, but particularly if there has been no correction yet made, pet should consider here the possibility of a simple phrase error. Examples are word doubling and omission of a common function word.

\section{Various Extensions}

Damerau transformations involving the space character (e.g. splitting a word) have not been implemented yet. Handling deletion of a space, or substitution of another character for a space, are straightforward additions to the morphological process. Transposition of a space could be dealt with by setting up an expectation upon discovering deletion of the last character of a word that the 'deleted' character may be attached to the beginning of the next word. Addition of a space is trickier because of the focus on the word as a processing unit, e.g. corrections for "the re" 
could include "there" or "the red", but the present system will not generate the former possibility.

At present the word in focus is always the newest word in the purview. Altering this would provide some right hand context information, which would among other things facilitate handling space addition. Allowing this change would necessitate a more complex backtracking mechanism, as there would be a focus lag between morphological processing and later phases.

It would be sensible to keep a reference to the wider context, i.e. be able to refer to earlier detections/corrections. With respect to the editor that pet is attached to, this could correspond to a log of errors already encountered in the file being edited. A recent Microsoft product ${ }^{2}$ keeps a record of personal habitual mistakes. Either could be a valuable aid in choosing the correct correction.

The system could possibly make better use of the graph state of its lexicon. Word transformation implies either implicit or explicit string comparison. The advantage of a graph over a trie is that it allows for comparison from the end of the word and well as the beginning.

\section{Testing and Evaluation}

With the aim of evaluating the effectiveness of shallow processing, tests will be carried out to see what proportion of different types of errors can be dealt with elegantly, adequately and/or efficiently. Under examination will be the number of errors missed/caught and wrongly/rightly corrected. Different components and configurations of the system will be compared, for example the error rules v. p.b.t.'s. Parameters of the system will be varied, for example the breadth of the purview, the position of the purview focus, the number of correction candidates and the timing of their generation. Will shallow processing miss too many of the errors cooperative error processing is aimed at?

There are two significant difficulties with collecting test data. The central difficulty is finding a representative sample of genuine errors by native speakers, in context, with the correct version of the text attached. Apart from anything else, 'representative' is hard to decide - spectrum of errors or distribution of errors? Secondly, any corpus of text usually contains only those errors that were left undetected in the text. Cooperative processing deals with errors that one backtracks to catch; if not a different class or range, these at least might have a different distribution of error types.

The ideal data would be records of peoples' keystrokes when interacting with an editor while creating or editing a piece of text. This would

\footnotetext{
${ }^{2}$ Microsoft Word 6.0 Autocorrect Wizard
}

allow one measure of the (linguistic) feasibility of cooperative error processing: the effectiveness of shallow processing over errors revealed by the keystroke-record data. There does not appear to be an English source of this kind, so it is planned to compile one.

For comparison, a variety of other data has been collected. Preliminary tests used generated errors, from a program that produces random Damerau slips according to an observed distribution (Pollock, 1983), using confusion matrices where appropriate (Kernighan, 1990). Assembled data includes the Birkbeck corpus (Mitton, 1986) and multifarious misspelling lists (without context). Suggestions have been made to look for low frequency words in corpora and news/mail archives, and to the Longmans learner corpus (not native speakers).

\section{Acknowledgements}

Thanks to all who offered advice on finding data, and to Doug Mcllroy, Sue Blackwell and Neil Rowe for sending me their misspelling lists.

This work is supported by a British Telecom Scholarship, administered by the Cambridge Commonwealth Trust in conjunction with the Foreign and Commonwealth Office.

\section{References}

Hiyan Alshawi. 1992. The Core Language Engine. Cambridge, Massachusetts: The MIT Press.

Fred J. Damerau. 1964. "A Technique for Computer Detection and Correction of Spelling Errors",

Roger Garside, Geoffrey Leech and Geoffrey Sampson, eds. 1987. The Computational Analysis of English. Longman. Commun. ACM, $7(3): 171-176$.

Claire Grover, John Carroll and Ted Briscoe. 1993. "The Alvey Natural Language Tools Grammar (4th Release)", Tech. Rep. 284, Computer Lab, University of Cambridge.

Mark D. Kernighan, Kenneth W. Church and William A. Gale. 1990. "A Spelling Correction Program Based on a Noisy Channel Model", Proc. Coling-90, pp 205-210.

Roger Mitton, ed. 1986. A Collection of Computer-Readable Corpora of English Spelling Errors (ver. 2). Birkbeck College, University of London.

Joseph J. Pollock and Antonio Zamora. 1983. "Collection and Characterization of Spelling Errors in Scientific and Scholarly Text", J. Am. Soc. Inf. Sci., 34(1):51-58. 
Stephen G. Pulman and Mark R. Hepple. 1993. "A feature-based formalism for two-level phonology: a description and implementation", Computer Speech and Language, 7(4):333-358.

Edward M. Riseman and Allen R. Hanson. 1974. "A Contextual Postprocessing System for Error Correction Using Binary $n$-Grams", IEEE Trans. Comput., C-23(5):480-493. 